\title{
Transient optical response of a single gold nanoantenna: the role of plasmon detuning
}

\author{
M. Zavelani-Rossi ${ }^{1,2}$, D. Polli ${ }^{1,2}$, S. Kochtcheev ${ }^{3}$, A.-L. Baudrion ${ }^{3}$,
} J. Béal ${ }^{3}$, V. Kumar ${ }^{1}$, E. Molotokaite ${ }^{1}$, M. Marangoni ${ }^{1,2}$, S. Longhi ${ }^{1,2}$, G. Cerullo ${ }^{1,2}$, P.-M. Adam ${ }^{3, *}$ and G. Della Valle ${ }^{1,2, *}$

${ }^{1}$ Dipartimento di Fisica, Politecnico di Milano, Piazza L. da Vinci 32, I-20133 Milano, Italy

${ }^{2}$ Istituto di Fotonica e Nanotecnologie, Consiglio Nazionale delle Ricerche, Piazza L. da Vinci 32, I-20133 Milano, Italy

${ }^{3}$ Laboratoire de Nanotechnologies et d'instrumentation Optique, Institut Charles Delaunay UMR CNRS 6279, Université de Technologie de Troyes, 12 Rue Marie Curie, CS 42060, 10004 Troyes Cedex, France

E-mail: pierre_michel.adam@utt.fr, giuseppe.dellavalle@polimi.it

\begin{abstract}
We study by femtosecond pump-probe microscopy the transient plasmonic response of individual gold nanoantennas fabricated by electron-beam lithography on a glass substrate. By exploiting the capability of the fabrication technique to control geometrical parameters at the nanoscale, we tuned the plasmonic resonance in a broad wavelength range, from the visible to the infrared. Numerical simulations based on a three-temperature model (3TM) for the electrons and lattice dynamics, combined with
\end{abstract}

\footnotetext{
*To whom correspondence should be addressed
} 
full-wave numerical analysis and semiclassical theory of optical transitions in the solid state, are compared with the measurements on a single gold nanoantenna probed at different wavelengths. The agreement between the experiment and the prediction of the 3TM turns out to be comparable to that achievable with the more sophisticated Boltzmann equation formalism. We also investigate the influence of the plasmon detuning with respect to the pump and probe wavelengths on the nonlinear optical response using different nanoantennas. Quantitative comparison of the experimental data with the theoretical model also provides a disentanglement of the different contributions to the optical nonlinearity of gold giving rise to the complex features observed in the transient optical response. Our study provides a complete analysis of the physical mechanisms dominating the nonlinear plasmon dynamics of an individual nanoobject taking place on a few ps time scale.

\section{Keywords}

Plasmonics, Nanoantennas, Ultrafast spectroscopy, Nanooptics, Nonlinear optics

Since more than a decade, plasmonics has emerged as a new branch of physics with numerous applications, under way or considered ${ }^{1,2}$. It covers a broad range of fields such as optoelectronics with new sources, detectors, modulators and waveguides ${ }^{3}$, biology and medicine with novel biosensors ${ }^{4,5}$ and therapies ${ }^{6,7}$, renewable energies with functionalized materials for photovoltaics ${ }^{8}$ and photocatalysis $^{9}$, just to mention a few. A very recent novel branch of plasmonics is aimed at the investigation of the nonlinear optical phenomena taking place in metal nanostructures ${ }^{10}$. Noble metals are well known to exhibit giant optical nonlinearities ${ }^{11,12}$, but ohmic losses have prevented any practical exploitation of bulk metals as optical media, and the potential of gold and silver as nonlinear materials has been overlooked for many decades. Contrary to bulk media, nanostructured metallic materials displaying plasmonic resonances can become highly transparent in certain frequency intervals and are thus good candidates for the development of a new generation of nonlinear 
devices with potential application to all-optical switching ${ }^{13-15}$ and sensing ${ }^{16}$. To address the challenging task of designing nonlinear nanoplasmonic devices, a quantitative understanding and modeling of the nonlinear optical phenomena taking place in metallic nanostructures is mandatory.

From a more fundamental point of view, surface plasmons are elementary excitations where photons are coupled to free electrons ${ }^{17}$. As such, both optics at the nanoscale and solid state physics are required to fully understand their nonlinear properties, arising from strong-field excitation of conduction electrons and subsequent relaxation dynamics, taking place on the femtosecond and picosecond timescales (see e.g. Refs. 18-20 and references therein). Local spectroscopy on individual nano-objects is the most direct and effective approach to achieve a clearcut evidence of all the phenomena involved, without incurring in inhomogeneity and averaging effects present in ensembles, only leading to partial results that would preclude a precise modeling. This is especially true when considering elongated nanostructures and in particular nanoantennas ${ }^{21}$ that exhibit strong polarization dependent response which would be washed out in an ensemble of dispersed nano-objects. To study the nonlinear optical response of a single nano-object, one should combine transient non-linear spectroscopy with spatially resolved microscopy, by coupling a femtosecond pump-probe set-up to a confocal microscope. It is worth noting that, contrary to the case of the long timescale optical response (related to acoustic oscillations of the nanostructure) which has been extensively investigated in many papers (see e.g. Refs. 20,22 and references therein), the ultrafast dynamics of a single plasmonic nanoobject (related to electron thermalization processes) has received much less attention. To the best of our knowledge, there's only a very limited number of papers reporting on such an investigation, mostly probing the dynamics at a single wavelength ${ }^{23,24}$, and only one providing a broad-band investigation but limited to a very small nanostructure (a gold nanorod of $12 \mathrm{~nm}$ diameter and about $40 \mathrm{~nm}$ length, having the fundamental plasmonic resonance at around $800 \mathrm{~nm}^{25}$ ). 
We report here an extended experimental study on the transient plasmonic response of a set of single gold antennas of different size, spanning a very broad range of plasmonic resonances, from the visible to the infrared. The fundamental resonance of the nanoantennas is tuned thanks to the control of the nanorod shape afforded by the Electron Beam Lithography (EBL) nanofabrication technique. We developed an ad hoc spatially resolved two-colour pump-probe setup allowing to tune the probe wavelength independently from that of the pump. Different regimes of the transient response, depending on the spectral tuning/detuning of the plasmon resonance of the nanoantenna with respect to the pump and probe wavelengths, were revealed. A three-temperature model (3TM) is employed to quantitatively describe the dynamics of the energy degrees of freedom of the nano-system after impulsive photo-excitation, without recurring to the Boltzmann equations formalism. This simpler approach, combined with a semi-classical modeling of optical transitions in gold and finite-element-method (FEM) numerical simulations of the electromagnetic response of the antennas, allowed us to reproduce all the features observed in the experiments and thus to provide a clearcut explanation of the different dynamics experimentally observed at the nanoscale.

\section{Experimental results}

We first investigated the linear optical response of isolated nanoantennas. Fig. 1 shows the experimental scattering spectra of three nanoantennas with nominal lengths $L=160 \mathrm{~nm}$, $170 \mathrm{~nm}$ and $190 \mathrm{~nm}$, recorded by dark-field nano-spectroscopy with unpolarized light. The actual lengths, estimated from SEM images (insets in Fig. 1), turned out to be $L_{e} \simeq 157$ $\mathrm{nm}, 168 \mathrm{~nm}$ and $188 \mathrm{~nm}$, and thus in good agreement with the nominal values. The peak of the spectra corresponds to the excitation of the longitudinal plasmonic resonance of the nanoantenna, whose spectral position is estimated to be $\lambda_{R 1}=850 \mathrm{~nm}, \lambda_{R 2}=895 \mathrm{~nm}$, and $\lambda_{R 3}=925 \mathrm{~nm}$, for the three nanoantennas of Fig. 1. The experimental spectra are compared 

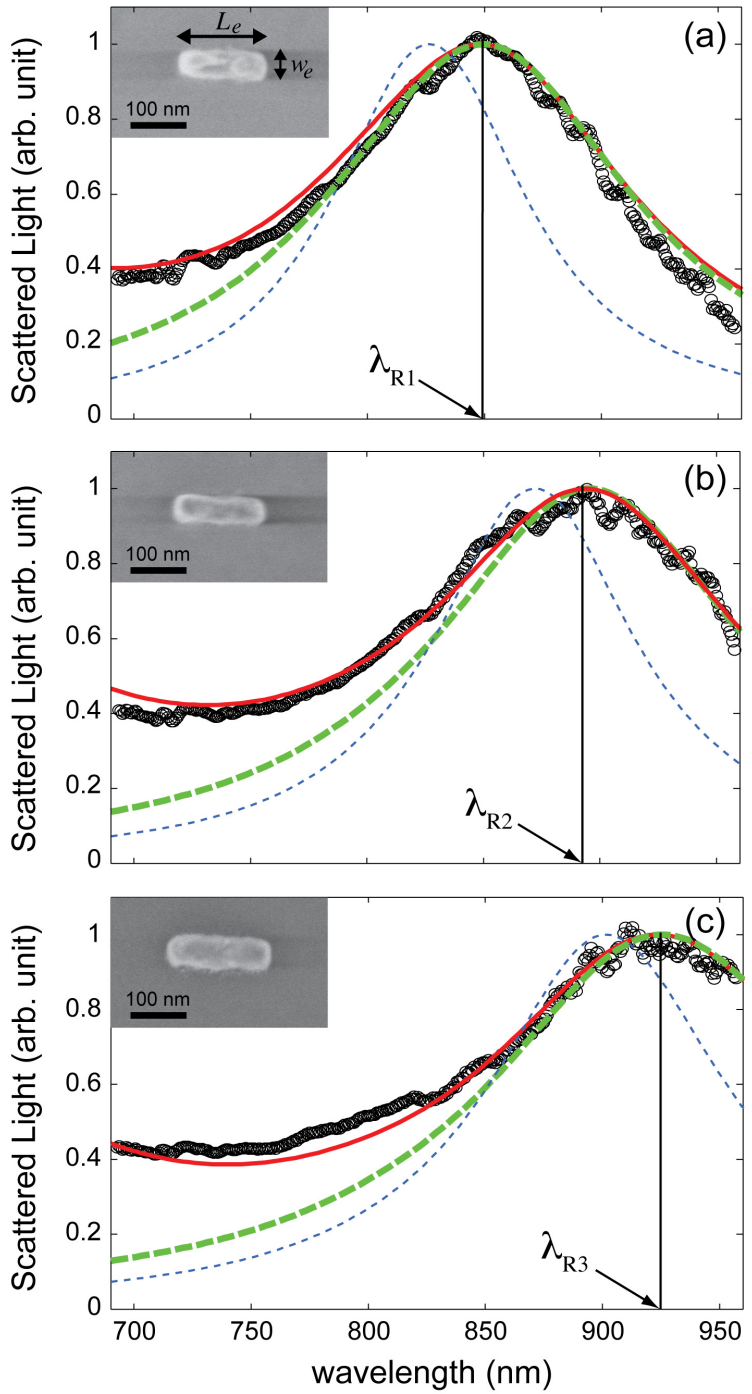

Figure 1: Linear scattering spectra of single gold nanoantennas of different size: (a) $L_{e}(L)=$ $157(160) \mathrm{nm}, w_{e}(w)=51(55) \mathrm{nm},\left(\right.$ b) $L_{e}(L)=168(170) \mathrm{nm}, w_{e}(w)=48(55) \mathrm{nm}$, and $(\mathrm{c})$ $L_{e}(L)=188(190) \mathrm{nm}, w_{e}(w)=62(65) \mathrm{nm} . L_{e}$ and $w_{e}$ are the length and width estimated from SEM images (see inset), where $L$ and $w$ are the values employed in the numerical simulation to retrieve the best fit of the experimental spectra. The unpolarized dark-field measurement (black circles) is compared with the theoretical scattering spectrum for incident light polarized along the major axis of the nanoantenna with (dashed green curve) and without (blue dotted curve) modeling of the Cr adhesion layer. The solid red curve also considers the contribution arising from the short-axis plasmonic resonance, computed under excitation with light polarized along the minor axis of the nanoantenna.

with numerical simulation of the scattering spectra based on FEM analysis (see Appendix I for details). In the simulations we assumed, for the naonantenna length, the nominal value $L$, whereas the nanoantenna width $w$ was a fitting parameter. A very good agreement with 
the measurements, both in terms of spectral position and width of the plasmonic peak, was achieved for values of $w$ a bit larger than the actual values $w_{e}$ for all the three nanoantennas (Fig. 1). This can be explained by noting that $\lambda_{R}$ basically scales linearly with $L$, but $w$ also has an impact on the plasmonic peak, shifting $\lambda_{R}$ towards the blue (red) for larger (shorter) $w$, similarly to the case of cylindrical nanoantennas (see e.g. Ref. 26). Since for the present antennas $L_{e}$ is slightly shorter than $L$, in order to retrieve the actual $\lambda_{R}$, in the simulations $w$ ought to be larger than $w_{e}$. It is also worth noting that we found a substantial improvement in the accuracy of the numerical analysis when taking into account the 3-nm thin Cr layer employed to increase the adhesion of the gold nanoantennas on top of the glass substrate. The agreement between experimental and theoretical scattering spectra demonstrates the capability of the EBL technique to achieve a reliable control of the geometrical parameters of the nanoantennas, and, at the same time, the capability of the numerical modeling to retrieve a correct quantitative prediction of their linear plasmonic response. These results will be the basis of our systematic investigation of the role played by plasmon detuning in the transient optical response of single gold nanoantennas excited by ultrafast laser pulses.

The ultrafast studies were divided in two sets of experiments. In a first set, we used a setup analogous to the one used in Ref. 25 but with a broadband tunability of the probe beam and an independently tunable pump beam (so we could change the probe wavelength keeping fixed the pump one). We selected a nanoantenna of 170-nm length and 50-nm width [Fig. 2(a)]. The simulated absorption, total scattering and extinction cross-section spectra of this nanoantenna are shown in Fig. 2(b), together with the experimental normalized scattering spectrum (black circles). The scattering peak is located at $902 \mathrm{~nm}$, whereas the absorption peak is slightly red-shifted (by about $7 \mathrm{~nm}$ ), in agreement with what reported in previous papers on gold nanoatennas of comparable size ${ }^{27}$. The extinction cross-section (the sum of the two spectra) turns out to be peaked at $904 \mathrm{~nm}$, that we can take as a good estimation of the fundamental dipolar plasmonic resonance wavelength $\lambda_{R}$ of the nanoantenna. We pumped the antenna off-resonance at $\lambda_{P}=780 \mathrm{~nm}$. To investigate the ultrafast 
dynamics of this plasmonic resonance, we tuned the probe wavelength in the range 850-1000 $n m$ and then recorded, as a function of the pump-probe time delay $t$, the differential transmission $\Delta T / T=\left(T_{o n}-T_{o f f}\right) / T_{o f f}$, where $T_{o n}$ and $T_{\text {off }}$ are the probe signals transmitted by the excited and unperturbed nanoantenna, respectively. Results are shown in Fig. 2(c) for
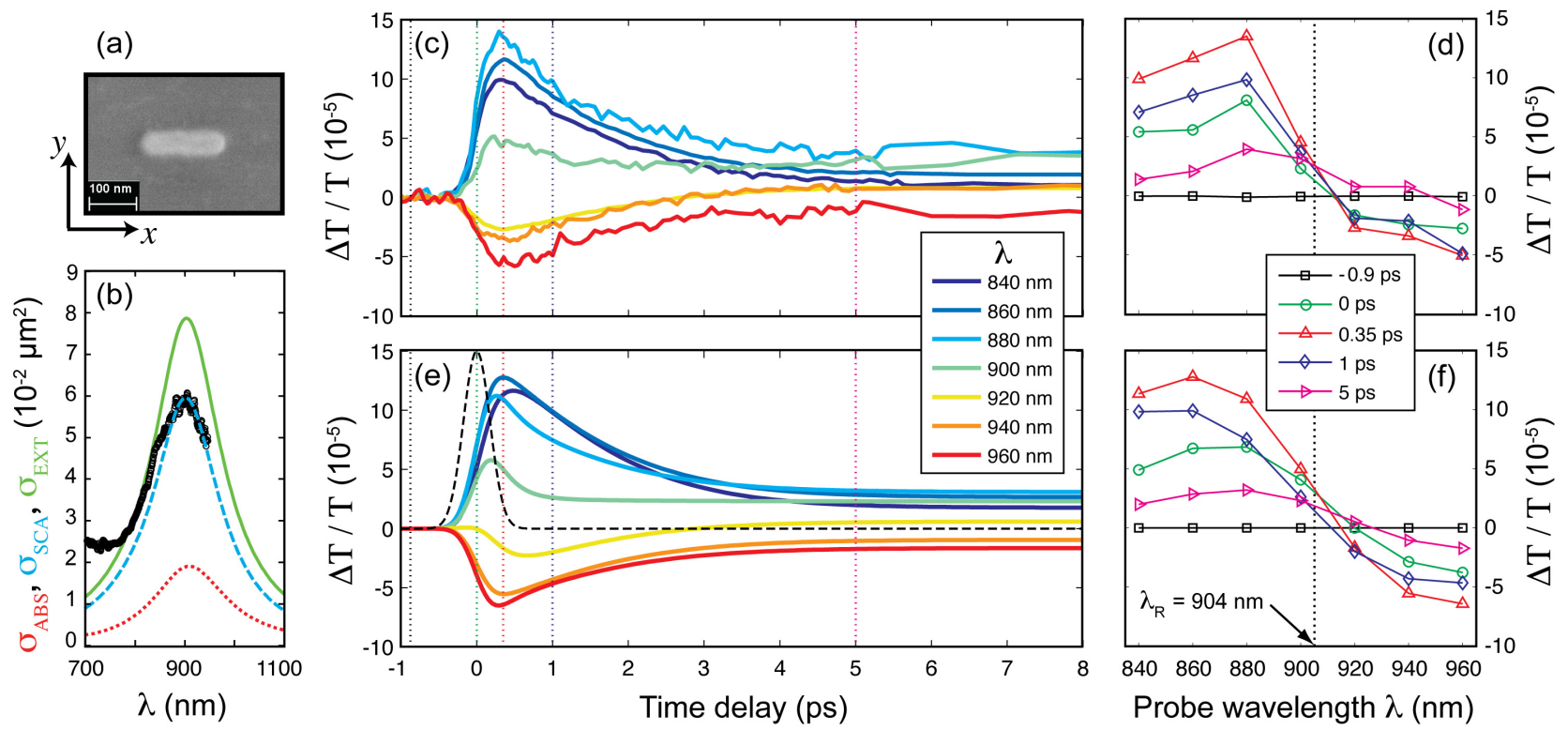

Figure 2: (a) SEM image of a gold nanoantenna of 170-nm length and 50-nm width. (b) Theoretical absorption (red dotted curve), total scattering (blue dashed curve) and extinction (green solid curve) cross-sections for $x$-polarized light [cf. Appendix I for details]. Black circles represent the normalized scattering spectrum recorded by dark-field measurements with unpolarized light. (c) Experimental and (e) theoretical $\Delta T / T$ time traces for the nanoantenna in (a) as a function of time delay and for different probe wavelengths pumped at $780 \mathrm{~nm}$. Dashed curve in (e) is the pump-probe cross-correlation assumed in our simulations [corresponding to the dynamics of $P_{a}(t)$ in Eq. (S6)]. (d) Experimental and (f) theoretical $\Delta T / T$ spectra, obtained from the dynamics in (c) and (e) respectively.

$65 \mu \mathrm{J} / \mathrm{cm}^{2}$ incident pump fluence. Note that both positive and negative signals are observed, depending on the probe wavelength. All the signals display a resolution-limited rise peaking at around 0.3 ps delay, after which a monotonic decrease (in modulus) is observed at all wavelengths until a long-living plateau is established, after about 5 ps. Fig. 2(d) shows the experimental $\Delta T / T$ spectra at different pump-probe delays, retrieved from the wavelengthdependent time traces. Basically, these spectra exhibit a derivative shape as compared to the typical extinction spectra in continuous-wave regime, which are almost Lorentzian [Fig. 1 
and Fig. 2(b)]. However there are some features that do not correspond to a purely derivative behavior: i) the crossing of the zero (the socalled isosbestic point) is located at around $910 \mathrm{~nm}$, i.e. slightly red-shifted as compared to $\lambda_{R}$; ii) the wing at shorter wavelengths is more pronounced; iii) as the time delay increases, the derivative shape is more distorted and the isosbestic point is increasingly red-shifted. Finally, it is worth noting that in our experiment acoustic oscillations of the nanoantenna are observed on a longer time scale, as periodic oscillations of the $\Delta T / T$ signal with about 150 ps period. These oscillations are ascribable to the onset of the fundamental extensional mode of the nanonatenna and do not play a role on the 10 ps time scale under investigation (see Appendix IV in the SI for details).
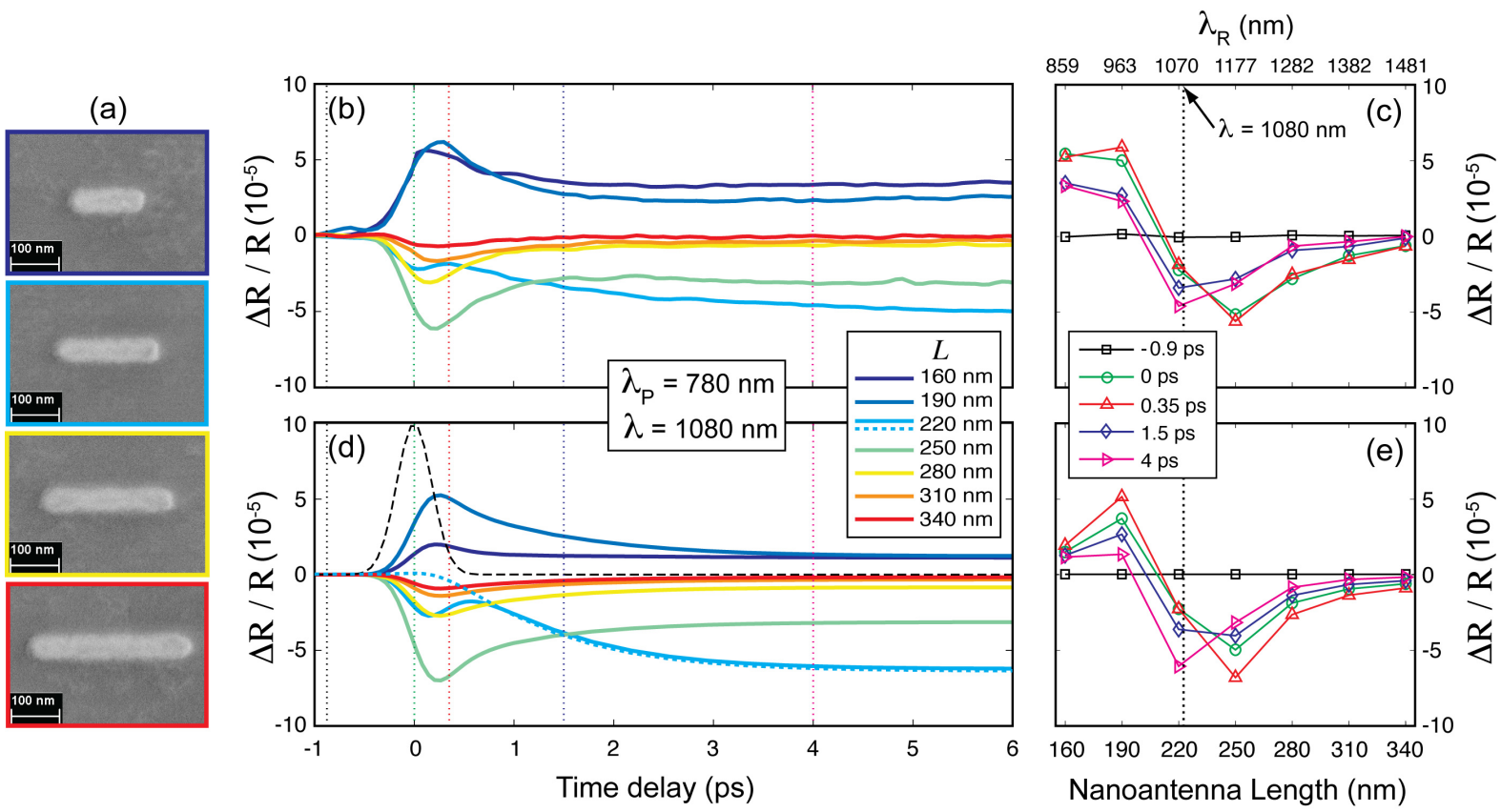

Figure 3: (a) SEM images of single gold nanoantennas of comparable width $w=50 \mathrm{~nm}$ and different length (from top to bottom): $160 \mathrm{~nm}, 220 \mathrm{~nm}, 280 \mathrm{~nm}, 340 \mathrm{~nm}$. (b) Experimental and (d) theoretical $\Delta R / R$ time traces at $\lambda=1080 \mathrm{~nm}$ and $\lambda_{P}=780 \mathrm{~nm}$ for nanoantennas of different length. Dotted curve in (d) represents the theoretical $\Delta R / R$ for the $220 \mathrm{~nm}$ long nanoantenna neglecting the contribution from non-thermal electrons. Dashed curve in (d) is the pump-probe cross-correlation assumed in our simulations [corresponding to the dynamics of $P_{a}(t)$ in Eq. (S6)]. (c) Experimental and (e) theoretical cross-sections of the dynamics in (b) and (d) respectively, at different time delays.

In a second set of experiments we decided to select a probe wavelength, corresponding 
to the resonance peak of a particular antenna and to characterize single nanoantennas of different lengths. To have a full set of measurements with the very same experimental conditions we decided to use the reflection geometry which is less complex and guarantees higher reproducibility, even though being more challenging on a single nanoobject because of the background noise due to substrate reflection. We selected as the probe wavelength 1080 $\mathrm{nm}$, corresponding to the peak resonance wavelength of the nanoantenna with $220 \mathrm{~nm}$ length, and characterized 6 individual nanoantennas with lengths from $160 \mathrm{~nm}$ to $340 \mathrm{~nm}$ [some of which are shown in Fig. 3(a)]. These nanoantennas have plasmonic resonances ranging from the visible to the near infrared, as detailed in Appendix I and Fig. S1(b)-(c). The differential reflection signal, that we measure, is defined, similarly to the differential transmission, as $\Delta R / R=\left(R_{o n}-R_{o f f}\right) / R_{o f f}, R_{o n}$ and $R_{o f f}$ being the probe signals reflected by the excited and unperturbed nanoantenna, respectively. Results are shown in Fig. 3(b) for $63 \mu \mathrm{J} / \mathrm{cm}^{2}$ incident pump fluence at $\lambda_{P}=780 \mathrm{~nm}$. Again, we observed both positive and negative signals, depending on the nanoantenna length. Similarly to the previous experiment, the signal displays resolution limited rise peaking at around $0.3 \mathrm{ps}$, after which a monotonic decrease (in modulus) is observed until a long-living plateau is established (after about 5 ps), for all the antennas except for the one which is in resonance with the probe (i.e. the one with $L=220 \mathrm{~nm}$ ). For this antenna a non-monotonic behaviour is observed, with a dip at $\sim 0.15$ ps followed by a peak at $\sim 0.45$ ps before the monotonic decrease to the long-living plateau, which also turns out to be particularly intense as compared to that observed for the other antennas. Fig. 3(c) shows the experimental $\Delta R / R$ signal as a function of the nanoantenna length for different pump-probe delays. Interestingly, these curves also exhibit a derivative behaviour, similar to the one observed in the $\Delta T / T$ spectra of the previous experiment. This suggests that the detuning of the plasmonic resonance with respect to the probe wavelength dominates the transient plasmonic response of a nanoantenna.

To understand the origin of the complex spectro-temporal features observed experimentally, and to elucidate the role played by the different physical processes involved in the 
transient optical response of individual plasmonic nanoatennas, we developed a quantitative model of the two experiments. This allows us also to compare the accuracy of our model (which is based on a three-temperature rate equations formalism) to the more sophisticated model (based on Boltzmann equation formalism) used in Ref. 25 (first set), and to validate (for the first time, to our knowledge) a model of the transient optical response of a single nanoobject in reflection (second set).

\section{Theoretical Model}

A sketch of the model used to describe the transient nano-plasmonic response is shown in Fig. 4(a). The pump pulse initially excites free electrons in a non-thermal distribution, with an excess energy per unit volume $N$ that is subsequently released, by electron-electron scattering, to the thermalized electronic population, heating it up to a higher temperature $T_{E}$. Then electron-phonon scattering causes the electronic gas to cool down and the lattice to increase its temperature $T_{L}$, until a long-living equilibrium temperature is achieved. A much slower phonon-phonon scattering process causes the excess energy originally delivered to the nanoobject to flow into the environment (placed at room temperature $T_{0}$ ). The weak probe pulse interacts with all the three different degrees of freedom of the system (non-thermal electrons, thermal electrons and the lattice) and the effects of such an interaction depend on the pump-probe time delay and the probe wavelength, giving rise to the rich scenario of dynamical features observed in the transient plasmonic response of our experiments (cf. Fig. 2 and Fig. 3). To quantitatively model such a response, either in transmission or in reflection, we followed a segregated approach allowing one: (i) to describe the temporal evolution of the three dynamical variables $N, T_{E}$ and $T_{L}$ driven by the incident pump pulse; (ii) to determine how these dynamical variables affect the nanoantenna medium, i.e. gold permittivity, in a broad range of wavelengths; (iii) to compute the subsequent modifications induced in the plasmonic response of the antenna to the weak probe pulse. 

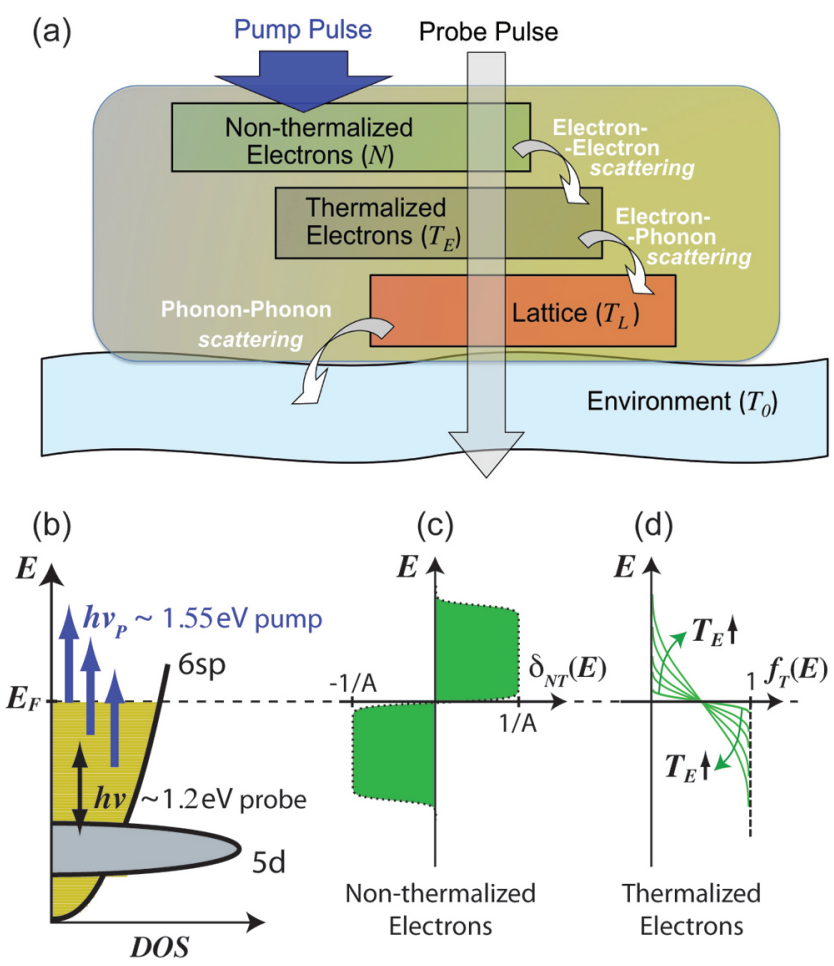

Figure 4: (a) Sketch of the 3TM for energy relaxation in a nanoantenna. (b) Drawing of gold density of states showing intraband pump absorption (blue arrows) and interband probe optical transitions (black arrow). (c) Normalized pump-induced variation of the energy occupation probability of non-thermalized electrons (see Appendix III.B in the SI for details). (d) Smearing of the Fermi-Dirac function for increasing temperature responsible to the modulation of occupation probability of thermalized electrons.

Issue (i) is addressed by the so-called Three-Temperature Model (3TM) that has been proposed and successfully exploited for thin gold films ${ }^{28,29}$ and plasmonic nanoparticle ensembles, from the pioneering works on gold nanoshells ${ }^{18}$ to most recent results on heavily-doped semiconductor nanocrystals ${ }^{30}$, (see Appendix III.A in the SI for details).

To address issue (ii), we followed an approach initiated in the late nineties with the pioneering work by Sun and coworkers on thin films in the visible ${ }^{28}$, recently validated by ultrafast pump-probe spectroscopy combining high temporal resolution $(\sim 15 \mathrm{fs})$ and broad spectral coverage $(490-560 \mathrm{~nm})^{31}$, and also extended to metal-dielectric multilayers in the near infrared (850-1100 nm) ${ }^{32}$. Such an approach has also led to the first formulation of the cubic nonlinearity of gold as a thermo-modulational nonlinearity ${ }^{29,33}$. The three variables $N$, $T_{E}$ and $T_{L}$, whose dynamics is calculated by the $3 \mathrm{TM}$, induce different contributions to the 
transient gold permittivity: the variables $N$ and $T_{E}$ affect the interband permittivity due to bound electrons, whereas $T_{L}$ affects the intraband permittivity due to free electrons in the conduction band (so-called Drude-Sommerfeld permittivity). More precisely, the temporal variations of $N$ and $T_{E}$ translate into a temporal variation of the occupation probability of non-thermalized (NT) and thermalized (T) electrons, respectively given by ${ }^{28}$ :

$$
\begin{aligned}
\Delta f_{N T}(E, t) & =\delta_{N T}(E) N(t), \\
\Delta f_{T}(E, t) & =f_{0}\left[E, T_{E}(t)\right]-f_{0}\left(E, T_{0}\right),
\end{aligned}
$$

being $f_{0}$ the Fermi-Dirac function, $E$ the electron energy (measured from the Fermi level) and $\delta_{N T}(E)$ a typical double steplike distribution ${ }^{28}$ [see Fig. 4(c)-(d), and Appendix III.B in the SI for further details]. A variation of the electron energy distribution due to thermal or non-thermal electrons results in a reduction (increase) of the occupation probability of the electronic states below (above) the Fermi energy, with increased (decreased) absorption for transitions involving final electronic states below (above) $E_{F}$ [Fig. 4(c)-(d)]. Quantitatively, such variations of absorption, or (equivalently) of the imaginary part of gold dielectric function $\Delta \epsilon_{N T(T)}^{\prime \prime}(\lambda, t)$ can be straightforwardly determined from gold band structure and semiclassical modeling of optical transitions in the solid state (as detailed in Appendix III.B). Then, the variation of the real part of the inter-band dielectric function $\Delta \varepsilon_{N T(T)}^{\prime}(\lambda, t)$ is retrieved from $\Delta \varepsilon_{N T(T)}^{\prime \prime}(\lambda, t)$ by Kramers-Kronig transformation.

As for the effect of lattice temperature $T_{L}$, there are two mechanisms to consider according to the Drude-Sommerfeld model of the free electrons permittivity (see e.g. Refs. 32,34 and references therein). An increase in the lattice temperature causes both an increase of the Drude damping $\Gamma$ (because of the increased electron-phonon scattering rate with temperature) and a decrease of the plasma frequency $\omega_{P}$ (caused by thermal expansion of the nanoantenna and subsequent decrease of the free carrier density $n$, which is related to the plasma frequency by $\omega_{P} \propto \sqrt{n}$ ). These two mechanisms give rise to two corresponding vari- 
ations of gold dielectric permittivity, $\Delta \epsilon_{L 1(2)}(\lambda, t)$, whose expressions are given in Appendix III.B.

The final step [point (iii)] of the modelling, that is the computation of plasmon dynamics induced by modulation of gold permittivity, is addressed by resorting to FEM numerical analysis. Actually, with the total variation of $\mathrm{Au}$ (complex) permittivity $\Delta \epsilon_{A u}(\lambda, t)=$ $\Delta \epsilon_{A u}^{\prime}(\lambda, t)+i \Delta \epsilon_{A u}^{\prime \prime}(\lambda, t)=\Delta \epsilon_{N T}(\lambda, t)+\Delta \epsilon_{T}(\lambda, t)+\Delta \epsilon_{L 1}(\lambda, t)+\Delta \epsilon_{L 2}(\lambda, t)$ at hand, the variation of nanoantenna cross-section $\Delta \sigma(\lambda, t)$ (being it either an absorption or a total scattering cross-section) is computed perturbatively as:

$$
\Delta \sigma(\lambda, t)=f_{1}(\lambda) \Delta \epsilon_{A u}^{\prime}(\lambda, t)+f_{2}(\lambda) \Delta \epsilon_{A u}^{\prime \prime}(\lambda, t)
$$

where

$$
\begin{aligned}
& f_{1}(\lambda)=\frac{d \sigma}{d \epsilon^{\prime}} \simeq \frac{\sigma\left[\lambda ; \epsilon_{A u}(\lambda)+\Delta \epsilon^{\prime}\right]-\sigma\left[\lambda ; \epsilon_{A u}(\lambda)\right]}{\Delta \epsilon^{\prime}} \\
& f_{2}(\lambda)=\frac{d \sigma}{d \epsilon^{\prime \prime}} \simeq \frac{\sigma\left[\lambda ; \epsilon_{A u}(\lambda)+i \Delta \epsilon^{\prime \prime}\right]-\sigma\left[\lambda ; \epsilon_{A u}(\lambda)\right]}{\Delta \epsilon^{\prime \prime}}
\end{aligned}
$$

In Equations (4)-(5), the cross-section $\sigma$ is determined by standard FEM numerical simulations [see Appendix I for details] and $\Delta \epsilon^{\prime}$ and $\Delta \epsilon^{\prime \prime}$ are the real and imaginary part of the average variation of $\mathrm{Au}$ permittivity induced by pump absorption in the considered nanoantenna, estimated according to the $3 \mathrm{TM}$ and the model of the cubic nonlinearity of gold above described.

To model the first set of pump-probe experiments, performed in transmission on the nanoantenna of $170 \mathrm{~nm}$ length and for different probe wavelengths, we computed the theoretical differential transmission as a function of the probe wavelength and time delay, according to the formula (in agreement with Refs. 24,25):

$$
\frac{\Delta T}{T}(\lambda, t)=-\frac{\Delta \sigma_{E X T}(\lambda, t)}{A}
$$


where $\sigma_{E X T}$ is the extinction cross-section (sum of the absorption and total scattering crosssections of the nanoantenna, see Appendix I for details), and $A=\pi w_{p r}^{2}, w_{p r}$ being the spot size of the probe beam. Results of the numerical simulations are reported in Fig. 2(e)-(f) for better comparison with the experimental data of Fig. 2(c)-(d). To model the second set of experiments, performed in reflection for a set of different nanoantennas at a given probe wavelength $\bar{\lambda} \simeq 1080 \mathrm{~nm}$, we computed the theoretical differential reflectivity as a function of the time delay as (see Appendix III.C for details):

$$
\frac{\Delta R}{R}(\bar{\lambda}, t)=\xi \frac{\Delta \sigma_{S C A}^{+}(\bar{\lambda}, t)}{\sigma_{S U B}}
$$

where $\sigma_{S C A}^{+}$is the backward total scattering cross-section of the nanoantenna (see Appendix II for details) and $\sigma_{S U B}=R_{S U B} A$, with $R_{S U B} \simeq 0.034$ the Fresnel reflectivity from the substrate (i.e. the air-glass interface) at normal incidence and $\xi$ the fraction of total backscattered power that is collected by the microscope objective under use $(N A \simeq 0.75$ in our experiments). It is worth noting that since the scatterer is supported on glass, the scattering is not isotropic in the plane orthogonal to the major axis of the nanoantenna (as it would be for the dipolar scattering of a nanorod antenna embedded in a homogeneous environment), and becomes more directed towards the substrate. From FEM numerical analysis we estimated $\xi \simeq 0.35$ for a typical gold nanorod antenna on fused-silica substrate. Results of the numerical simulations are reported in Fig. 3(d)-(e) for the same excitation condition of the experiments.

\section{Discussion}

A comparison between Fig. 2(c) and Fig. 2(e) indicates that our modeling is capable of capturing the main features observed in the experimental differential transmission measurements performed on a single nanoantenna. Interestingly, the agreement is not only qualitative but also quantitative, and, in particular, the almost derivative behaviour exhibited by the tran- 
sient plasmonic spectra of Fig. 2(d) is well reproduced in Fig. 2(f), as well as the deviations from the exact derivative shape observed in the initial steps of the dynamics, like the asymmetry of the spectra and the slight red-shift of the isosbestic point with respect to $\lambda_{R}$. The derivative behavior can be explained also according to a simplified two-temperature model (2TM) as a result of a red-shift of the plasmonic resonance induced by a real, positive and weakly dispersive $\Delta \epsilon$ ascribable to thermalized electrons. However the 2TM completely disregards the contribution from non-thermalized carriers. On the contrary, our model, though being a rate equation model like the 2TM, takes into account non-thermalized electrons by means of the $N$ variable (i.e. a third effective temperature). As detailed in a recent paper ${ }^{32}$, non-thermalized electrons, being much more dispersed in energy as compared to thermalized ones [cf. Fig. 4(c) and (d)], contribute to the total modulation of gold permittivity in a rather distinct way, giving rise to a complex scenario where, on the short time scale, both real and imaginary $\Delta \epsilon$ (and with opposite dispersion) are present [cf. in particular Fig. 8(a) and (b) in Ref. 32]. The non zero imaginary $\Delta \epsilon$ from non-thermalized carriers thus causes a slight broadening of the plasmonic resonance that, combined with the derivative effect produced by thermalized carriers, results in the red-shift of the isosbestic observed at the very initial steps of the dynamics, in agreement with what already observed in Ref. 25. Also, note that the agreement between experiments and modeling is achieved not only for the onset of the signal peaks and dips, but also for the onset of the long-living plateau. On this longer time-scale the lattice dynamics contributes to the transient variation of $\Delta \epsilon$, giving an imaginary contribution related to the modulation of the Drude damping (see Appendix III.B) and thus causing major deviations to the derivative behavior in the transient spectra. However, the shift of the isosbestic point turned out to be larger as compared to the theoretical prediction [cf. crossing of zero for magenta curves in Fig. 2(d) and (f)]. This is in agreement with what reported in Ref. 25 and can be attributed to other effects taking place on the long time-scale, possibly related to the modulation of the plasma frequency caused by lattice mechanisms not taken into account here. Indeed, our model includes the 
dominating mechanism of plasma frequency modulation (in the adiabatic limit) giving rise to $\Delta \epsilon_{L 2}$, but this turned out to be not enough to reproduce the shift of the isosbestic point on the long time scale. Finally, it is worth noting that, as compared to previous studies on the modeling of pump-probe experiments in metal based structures, here we quantitatively refined the mechanism of Drude damping modulation, thus avoiding an overestimation of the $\Delta \epsilon_{L 1}$. Actually, recent papers have put in evidence how the Drude damping $\Gamma$ is dominated by surface and grain boundaries scattering or defect scattering rather than phonon scattering $\Gamma_{e-p h n}$, especially in nanostructures ${ }^{34,35}$. Since it is only phonon scattering that gives rise to significant temperature effects, a precise estimation of this mechanism is required to achieve a correct quantitative estimation of the transient optical response on the long time-scale. This was provided by fitting the Holstein expression for $\Gamma_{e-p h n}$ [see e.g. Eq.(1) in Ref. 36] with a linear formula [Eq. (S12) in Appendix III.B]. As a final note on our first experiment, we point out that the results of Fig. 2 clearly indicates that the accuracy of the simpler 3TM is comparable to that of the more sophisticated Boltzmann equation model, taking into account the energy dependence of the relaxation rate of non-thermalized electrons, exploited in Ref. 25 (further comparison between the 3TM and the prediction of more accurate models based on Boltzmann equation formalism is reported in Appendix II.C).

A comparison between Fig. 3(b) and Fig. 3(d) indicates that a good quantitative agreement between the experimental data and the model is retrieved also for the second experiment, even though with less fidelity for the shortest nanoantennas with $L=160$ and $L=190 \mathrm{~nm}$. It is worth noting that validation of the model on this second experiment, achieved at a fixed probe wavelength and on different nanoantennas, is more challenging for several reasons. First of all, the need of rearranging the focus alignment when moving from one nanoantenna to another can result in some deviations from the nominal excitation conditions (i.e. incident fluence) and the probe area $A$ [that affects the $\Delta R / R$ as detailed by Eq. (7)]. Also, fabrication tolerances (i.e. deviation of fabricated parameters as compared to nominal value) affects differently the nanoantennas, having a higher impact on the 

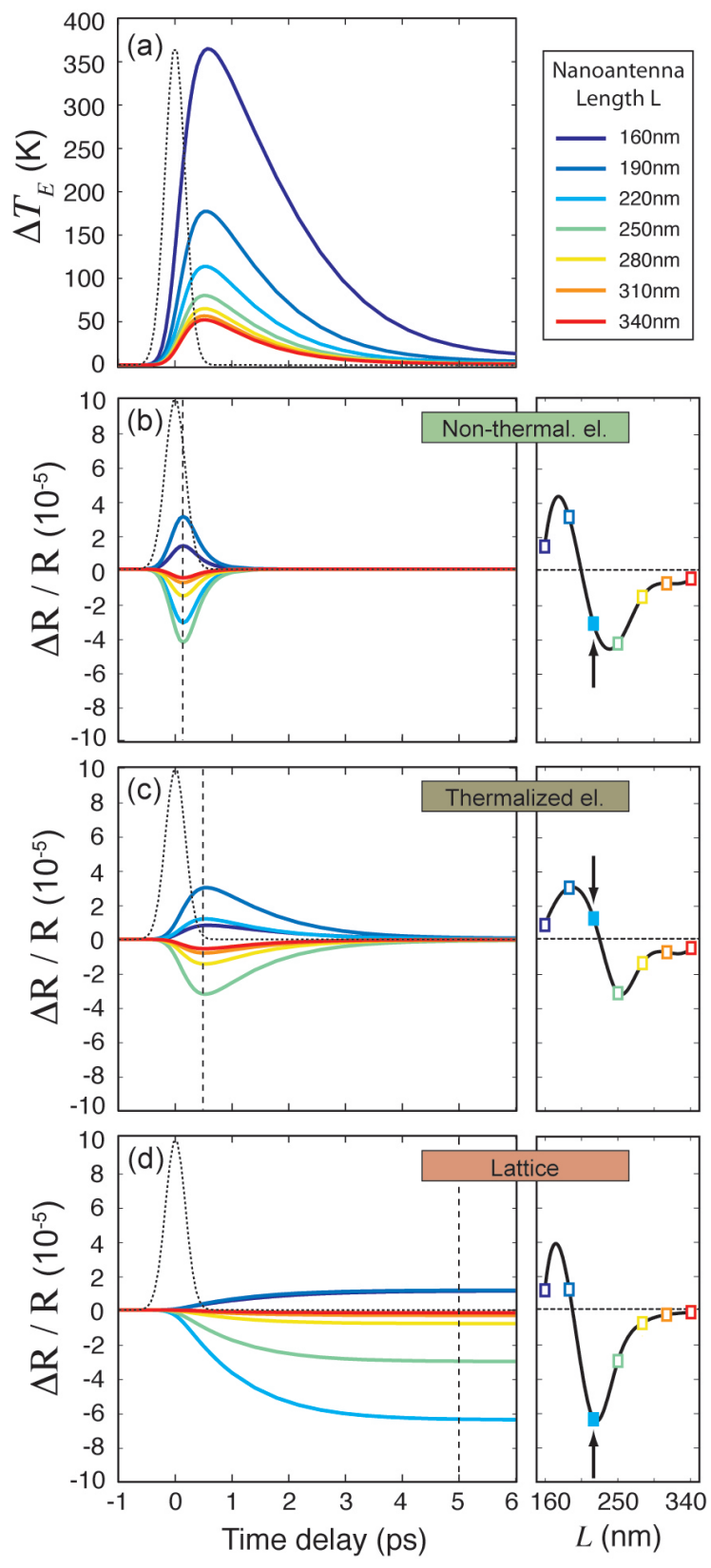

Figure 5: (a) Temporal dynamics of thermal electrons temperature in the different nanoantennas. (b)-(d) Disentanglement of the three contributions to the $\Delta R / R$ for the nanoantennas of Fig. 3 arising from: (b) non-thermalized electrons; (c) thermalized electrons; (d) lattice. Right panels in (b), (c) and (d) show the $\Delta R / R$ at the time delay indicated by vertical dashed lines in the corresponding left panels. Vertical arrows point out results for the nanoantenna with $220 \mathrm{~nm}$ length. Dotted curve is the pump-probe cross-correlation assumed in our simulations [corresponding to the dynamics of $P_{a}(t)$ in Eq. (S6)].

shortest ones (i.e. with $L=160$ and $190 \mathrm{~nm}$ ). Finally, and most importantly, the detuning between the probe and the peak plasmonic resonance $\Delta \lambda=\lambda-\lambda_{R}$ spans a much broader 
range of wavelengths, and also a broad range of pump detunings $\Delta \lambda_{P}=\lambda_{P}-\lambda_{R}$ is explored here. These latter circumstance implies that the operation conditions change a lot from one antenna to the other. This can be immediately realized when looking at the electronic temperatures achieved in the different nanoantennas, as estimated according to our modeling [Fig. 5(a)]. Note that the large difference in $\Delta \lambda_{P}$ translates into a large difference in terms of peak temperature variation, from about $50 \mathrm{~K}$ in the antenna with $L=340 \mathrm{~nm}$ to more than $350 \mathrm{~K}$ for the antenna with $L=160 \mathrm{~nm}$, with a monotonic trend from the longest antennas to the shortest ones. Considering all above issues, we can state that our modeling is quite predictive even for the second experiment, so it allows one to disentangle the three different contributions to the transient reflection signal arising from non-thermalized electrons, thermalized electrons, and the lattice (as the sum of the two mechanisms illustrated in previous section). These are shown in Fig. 5(b), (c) and (d), respectively. Note that since the dynamics of non-thermalized electrons is governed by $N$ whose relaxation time, according to the $3 \mathrm{TM}$ model is of the order of $260 \mathrm{fs}$ [estimated as the inverse of the electrons heating rate $a$ in Eq.(S3), see also Appendix III.C for details], the dynamics in Fig. 5(b) are much faster than those reported in Fig. 5(c), arising from thermalized electrons. However this is not the only difference between the two contributions to the total signal. Actually, depending on the length of the antenna, i.e. depending on the probe detuning $\Delta \lambda$, the $\Delta R / R$ from nonthermal electrons and the $\Delta R / R$ from thermal ones are both positive (negative) for negative (positive) detuning, but close to zero detuning they exhibit opposite signs. This happens for the antenna with $L=220 \mathrm{~nm}$, which is probed very close to resonance, and gives rise to a positive $\Delta R / R$ for non-thermal electrons and a negative $\Delta R / R$ from thermalized ones [cf. right panels in Fig. 5(b) and (c) for a clearer comparison]. Such a behaviour can be qualitatively understood by the following argument: for the present antenna, the contribution to the $\Delta R / R$ arising from the real $\Delta \epsilon$ (both from non-thermal and thermal electrons) is expected to be minimal, since the signal modulation caused by a shift of a resonance would be zero to the first order on the peak of the resonance (under perturbative excitation). This makes 
the contribution arising from non-thermalized electrons prominent, in light of the fact that they are also responsible for an imaginary $\Delta \epsilon$ extending to the infrared because of the broad range of electron energies involved into the creation of the out-of-equilibrium distribution $\delta_{N T}$ [cf. Fig. 4(b)]. Such a modulation causes a broadening of the resonance which in turns gives rise to a decrease of the peak reflectivity. Note that if one neglects the contribution from non-thermalized electrons [dotted line in Fig. 3(d)] the (dip-peak) oscillation observed in the initial part of the dynamics of the nanoantenna of $220 \mathrm{~nm}$ length is washed out. We conclude that such a behaviour is a fingerprint of the peculiar contribution provided by non-thermalized electrons, which can't be explained within a two-temperature model, but is well captured by a three-temperature dynamics without recurring to more complicated approaches based on the integration of Boltzmann equation for electrons thermalization.

\section{Conclusion}

We have performed a detailed experimental and theoretical investigation of the transient plasmonic response of individual gold nanoantennas fabricated on a glass substrate by EBL technique. The capability of EBL to control geometrical parameters and orientation of the nanoantennas, together with an ad hoc developed two-colour pump-probe microscopy setup, allowed us to study the influence of nanoantenna tunability on the rich spectral and temporal features observed in transient nanospectroscopy experiments. We found that the semiclassical model developed to describe the cubic optical nonlinearity of gold is capable of providing a quantitative description of the transient plasmonic response of individual gold nanoantennas on a broad range of wavelengths in the near infrared and for a broad range of plasmonic resonances. This model is validated for both transmission and reflection pumpprobe experiments with 300 fs time resolution, and it is capable of explaining the observed dynamics for both the two different time-scales of electron-electron and of electron-phonon relaxations. Most importantly, the accurate quantitative comparison between experiments 
and modeling allowed us to disentangle the different contributions to the transient plasmonic response arising from non-thermal electrons, thermal electrons and lattice dynamics. It is found that, contrary to what observed in the visible, in the near infrared and for in-resonance probing of the nanoantenna non-thermalized electrons can dominate the transient plasmonic response, giving rise to unexpected or even counter-intuitive features in the pump-probe signal. We believe that our results can definitely ascertain the validity of a thermal model for the cubic optical nonlinearity in gold and a quantitative approach to the modeling of nonlinear plasmonics based on a simple 3TM. This is of the highest importance in view of exploiting the capability of single plasmonic nanostructures and meta-surfaces to enhance optical nonlinearity of noble metals and to design novel plasmonic devices based on such phenomena. Further developments should consider an accurate investigation and modeling of the very initial part of the the plasmon dynamics (in the range of few tens of fs) where it is expected that some deviations from the semiclassical model can occur, in light of the presence of more subtle features that can't be disregarded on such a short timescale, like, e.g., plasmon dephasing and the energy dependence of electron-electron scattering rate. Also, the modeling of pump absorption ought to be refined by implementing saturation effects in order to address non perturbative regimes.

\section{Acknowledgments}

Financial support from the Fondazione Cariplo is acknowlegded by G.D.V., D.P., V.K., E.M. and S.L. through the project New Frontiers in Plasmonic Nanosensing (Grant No. 2011-0338), by M.Z.-R. through the project NANOCRYSLAS (Grant No. 2012-0824), and by M.M. through the project Surface-enhanced Coherent Antistokes Raman Scattering for label-free-sensitive detection (Grant No. 2012-0904). G.D.V., M.Z.-R. and P.-M.A. acknowledge COST Action MP1302-NanoSpectroscopy. P.-M.A. and S.K. would like to thank the platform Nano'mat. G.C. acknowledges support by the EC under Graphene Flagship (con- 
tract no. CNECT-ICT-604391).

\section{Methods}

Sample fabrication. Samples consisting of gold nanorods were fabricated by electron-beam lithography. Polymethylmethacrylate (PMMA) resist was deposited by spin-coating on a glass substrate. To reduce the influence on the beam deflection of the accumulated charges in the insulating substrate, a thin metallic layer was deposited on top of the PMMA prior to exposure and etched away afterwards, before development. The exposure was performed using a scanning electron microscope (SEM, S3500, Hitachi) equipped with a Nanometre Pattern Generation System (NPGS, J.C. Nabity Lithography Systems). After resist development, a $3 \mathrm{~nm}$ thick chromium adhesion layer and different gold layer thicknesses (in the range 30-50 nm) were deposited by electron-beam evaporation. The subsequent lift-off procedure was carried out in order to remove the unexposed resist.

Nano-spectroscopy. The linear scattering spectra of single nanorods have been recorded using a standard up-right microscope. The transmitted light, coming from a halogen lamp, is sent to a dark-field condenser (NA from 1.2 to 1.4) connected to the glass side of the sample via immersion oil. Note that with these illumination conditions, the nanorods are excited by evanescent waves produced by the total internal reflection of most visible wavelengths at the air-glass interface. The scattered light is collected with a high NA objective (0.95) and sent to a spectrometer (400-1000nm of spectral range) via a multimode fiber. The signal recorded on the glass substrate is then subtracted from the signal recorded on top of a nanorod and the rest is normalized by the lamp signal recorded in bright field, without the sample.

For the ultra-fast nano-spectroscopy experiments we have developed an ad hoc twocolour pump-probe setup. It starts with a mode-locked Er:fiber oscillator working at 40$\mathrm{MHz}$ repetition rate. The oscillator output is used to feed two independent synchronized Er-doped fiber amplifiers (EDFAs), each producing 350-mW average power, corresponding 
to $\sim 9 \mathrm{~nJ}$ per pulse, at a 1560-nm central wavelength. The EDFA outputs are compressed to nearly transform-limited sub-100-fs duration pulses by a silicon prism pair. One of the two EDFA outputs is frequency doubled by a 2 -mm-thick $\beta$-barium-borate (BBO) crystal cut for type-I phase matching $\left(\theta=21^{\circ}\right)$ to generate few-mW, 100-fs pump pulses at $780-\mathrm{nm}$. The other EDFA output is coupled to an highly non-linear fiber (HNLF) for spectral broadening. The HNLF generates a broadband supercontinuum (SC), whose dispersive wave extends to wavelengths as short as $820 \mathrm{~nm}$. The probe pulses are obtained by selecting a 10-nm portion of the SC by an interference filter in the 820-1100 nm wavelength range. Pump and probe pulses are synchronized by a computer-controlled delay line, collinearly combined by a dichroic beamsplitter and sent to a home-made microscope equipped with a high-numericalaperture $(\mathrm{NA})$ objective $[\mathrm{NA}=0.80]$. For transmission measurements the light is collimated by a long working distance objective $(\mathrm{NA}=0.75)$; for reflection measurements the light is collected by the focusing objective and separated from the incoming beam by a broadband beam splitter. In both cases the probe is spectrally selected by a second interference filter and focused onto a silicon photodiode. The pump beam is modulated acousto-optically at a $70-\mathrm{kHz}$ frequency, and a lock-in amplifier measures either the differential transmission $(\Delta T / T)$ or differential reflectivity $(\Delta R / R)$ of the probe as a function of pump-probe delay. Due to chromatic aberration of the objective, pump and probe have different focal planes: we chose to place the sample in the probe focus, so that the pump is slightly defocused, thus guaranteeing optimum spatial overlap and homogeneity of the excitation. Typical average power used are 1-3 $\mathrm{mW}$ for the pump and 1-100 $\mu \mathrm{W}$ for the probe. The sample is mounted on a piezo-electric stage with 1-nm positioning accuracy and $100 \mu \mathrm{m} \times 100 \mu \mathrm{m}$ scan range. A sketch of the system showing the independent tunability of the pump and probe pulses is reported in Appendix I in the SI. Both pump and probe beams are linearly polarized, with the electric field aligned to the long axis of the nanoantenna [i.e. the $x$ axis in Fig. 2(a)]. The overall spatial resolution of our experiments, measured by imaging an isolated gold nanoantenna, is about $1 \mu \mathrm{m}$ FWHM, which is significantly smaller than 
the particle separation. Temporal resolution, estimated as the FWHM of the pump-probe cross-correlation, was about 300 fs.

\section{Associated Content}

\section{Supporting Information Available}

Further details of the data acquisition are reported in Appendix I. Numerical computation of the static absorption and scattering cross-sections of a single nanoantenna is given in Appendix II. Details of the modeling of the transient plasmonic response are provided in Appendix III. This material is available free of charge via the Internet at http://pubs . acs.org/.

\section{References}

(1) Lal, S.; Link, S.; Halas, N. J. Nano-optics from sensing to waveguiding. Nat. Photonics 2007, 1, 641-648.

(2) Schuller, J. A.; Barnard, E. S.; Cai, W.; Jun, Y.; White, J. S.; l. Brongersma, M. Plasmonics for extreme light concentration and manipulation. Nat. Mater. 2010, 9, $193-204$.

(3) Gramotnev, D. K.; Bozhevolnyi, S. I. Plasmonics beyond the diffraction limit. Nat. Photonics 2010, 4, 83-91.

(4) Stewart, M. E.; Anderton, C. R.; Thompson, L. B.; Maria, J.; Gray, S. K.; Rogers, J. A.; Nuzzo, R. G. Nanostructured Plasmonic Sensors. Chem. Rev. 2008, 108, 494-521.

(5) Anker, J. N.; Hall, W. P.; Lyandres, O.; Shah, N. C.; Zhao, J.; Van Duyne, R. P. Biosensing with plasmonic nanosensors. Nat. Materials 2008, 7, 442-453. 
(6) Huang, X.; El-Sayed, I. H.; Qian, W.; El-Sayed, M. A. Cancer Cell Imaging and Photothermal Therapy in the Near-Infrared Region by Using Gold Nanorods. J. Am. Chem. Soc. 2006, 128, 2115-2120.

(7) Loo, C.; Lowery, A.; Halas, N.; West, J.; Drezek, R. Immunotargeted Nanoshells for Integrated Cancer Imaging and Therapy. Nano Lett. 2005, 5, 709-711.

(8) Atwater, H. A.; Polman, A. Plasmonics for improved photovoltaic devices. Nat. Materials 2010, 9, 205-213.

(9) Awazu, K.; Fujimaki, M.; Rockstuhl, C.; Tominaga, J.; Murakami, H.; Ohki, Y.; Yoshida, N.; Watanabe, T. A plasmonic photocatalyst consisting of silver nanoparticles embedded in titanium dioxide. J. Am. Chem. Soc. 2008, 130, 1676-1680.

(10) Kauranen, M.; Zayats, A. V. Nonlinear plasmonics. Nat. Photonics 2012, 6, 737-748.

(11) Smith, D. D.; Yoon, Y.; Boyd, R. W.; Campbell, J. K.; Baker, L. A.; Crooks, R. M.; George, M. z-scan measurement of the nonlinear absorption of a thin gold film. J. Appl. Phys. 1999, 86, 6200-6205.

(12) Rotenberg, N.; Bristow, A. D.; Pfeiffer, M.; Betz, M.; van Driel, H. M. Nonlinear absorption in Au films: Role of thermal effects. Phys. Rev. B 2007, 75, 155426-1-5.

(13) MacDonald, K. F.; Samson, Z. L.; Stockman, M. I.; Zheludev, N. I. Ultrafast Active Plasmonics. Nat. Photonics 2009, 3, 55-58.

(14) Ren, M.; Jia, B.; Ou, J.-Y.; Plum, E.; Zhang, J.; MacDonald, K. F.; Nikolaenko, A. E.; Xu, J.; Gu, M.; Zheludev, N. I. Nanostructured Plasmonic Medium for Terahertz Bandwidth All-Optical Switching. Adv. Mater. 2011, 23, 5540-5544.

(15) Wurtz, G. A.; Pollard, R.; Hendren, W.; Wiederrecht, G. P.; Gosztola, D. J.; Podolskiy, V. A.; Zayats, A. V. Designed ultrafast optical nonlinearity in a plasmonic nanorod metamaterial enhanced by nonlocality. Nat. Nanotechnol. 2011, 6, 107-111. 
(16) Stockman, M. I. Ultrafast nanoplasmonics under coherent control. New J. Phys. 2008, 10, 025031.

(17) Raether, H. Surface Plasmons, 1st ed.; Springer-Verlag: Berlin, 1986.

(18) Averitt, R. D.; Westcott, S. L.; Halas, N. J. Ultrafast electron dynamics in gold nanoshells. Phys. Rev. B 1998, 58, R10 203-R10 2006.

(19) Knappenberger, K. L.; Schwartzberg, A. M.; Dowgiallo, A.-M.; Lowman, C. A. Electronic Relaxation Dynamics in Isolated and Aggregated Hollow Gold Nanospheres. J. Am. Chem. Soc. 2009, 131, 13892-13893.

(20) Hartland, G. V. Optical Studies of Dynamics in Noble Metal Nanostructures. Chem. Rev. 2011, 111, 3858-3887.

(21) Biagioni, P.; Huang, J.-S.; Hecht, B. Nanoantennas for visible and infrared radiation. Rep. Prog. Phys. 2012, 75, 024402.

(22) Tchebotareva, A. L.; Ruijgrok, P. V.; Zijlstra, P.; Orrit, M. Probing the acoustic vibrations of single metal nano particles by ultrashort laser pulses. Laser and Photon. Rev. $2009,4,581-597$.

(23) Pelton, M.; Liu, M.; Park, S.; Scherer, N. F.; Guyot-Sionnest, P. Ultrafast resonant optical scattering from single gold nanorods: Large nonlinearities and plasmon saturation. Phys. Rev. B 2006, 73, 155419-1-155419-6.

(24) Muskens, O. L.; Del Fatti, N.; Vallée, F. Femtosecond Response of a Single Metal Nanoparticle. Nano Letters 2006, 6, 552-556.

(25) Baida, H.; Mongin, D.; Christofilos, D.; Bachelier, G.; Crut, A.; Maioli, P.; Del Fatti, N.; Vallée, F. Ultrafast Nonlinear Optical Response of a Single Gold Nanorod near Its Surface Plasmon Resonance. Phys. Rev. Lett. 2011, 107, 057402. 
(26) Novotny, L. Effective Wavelength Scaling for Optical Antennas. Phys. Rev. Lett. 2007, 98, 266802.

(27) Husnik, M.; Linden, S.; Diehl, R.; Niegemann, J.; Busch, K.; Wegener, M. Quantitative Experimental Determination of Scattering and Absorption Cross-Section Spectra of Individual Optical Metallic Nanoantennas. Phys. Rev. Lett. 2009, 109, 233902.

(28) Sun, C.-K.; Vallée, F.; Acioli, L. H.; Ippen, E. P.; Fujimoto, J. G. Femtosecond-tunable measurement of electron thermalization in gold. Phys. Rev. B 1994-II, 50, 1533715348.

(29) Conforti, M.; Della Valle, G. Derivation of third-order nonlinear susceptibility of thin metal films as a delayed optical response. Phys. Rev. B. 2012, 85, 245423-1-245423-4.

(30) Della Valle, G.; Scotognella, F.; Kandada, A. R. S.; Zavelani-Rossi, M.; Li, H.; Conforti, M.; Longhi, S.; Manna, L.; Lanzani, G.; Tassone, F. Ultrafast Optical Mapping of Nonlinear Plasmon Dynamics in $\mathrm{Cu}_{2-x}$ Se Nanoparticles. J. Phys. Chem. Lett. 2013, 4, 3337-3344.

(31) Della Valle, G.; Conforti, M.; Longhi, S.; Cerullo, G.; Brida, D. Real-time optical mapping of the dynamics of nonthermal electrons in thin gold films. Phys. Rev. B 2012, 86, 155139 .

(32) Dal Conte, S.; Conforti, M.; Petti, D.; Albisetti, E.; Longhi, S.; Bertacco, R.; De Angelis, C.; Cerullo, G.; Della Valle, G. Disentangling electrons and lattice nonlinear optical response in metal-dielectric Bragg filters. Phys. Rev. B 2014, 89, 125122.

(33) Marini, A.; Conforti, M.; Della Valle, G.; Lee, H.; Tran, T. X.; Chang, W.; Schmidt, M. A.; Longhi, S.; Russell, P. S. J.; Biancalana, F. Ultrafast nonlinear dynamics of surface plasmon polaritons in gold nanowires due to the intrinsic nonlinearity of metals. New. J. Phys. 2013, 15, 013033-1-013033-19. 
(34) Yeshchenko, O.; Bondarchuk, I.; Gurin, V.; Dmitruk, I.; Kotko, A. Temperature dependence of the surface plasmon resonance in gold nanoparticles. Surface Science 2013, $608,275-281$.

(35) Chen, K.-P.; Drachev, V. P.; Borneman, J. D.; Kildishev, A. V.; Shalaev, V. M. Drude Relaxation Rate in Grained Gold Nanoantennas. Nano Lett. 2010, 10, 916-922.

(36) McKay, J. A.; Rayne, J. A. Temperature dependence of the infrared absorptivity of the noble metals. Phys. Rev. B 1976, 13, 673-685. 


\section{Graphical TOC Entry}

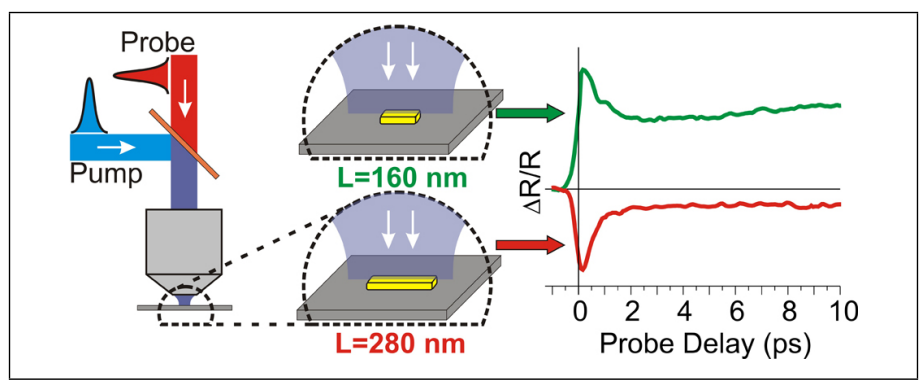

